\title{
COMPORTAMENTO ECOLOGICAMENTE CONSCIENTE DO CONSUMIDOR: ADAPTAÇÃO DA ESCALA ECCB PARA O CONTEXTO BRASILEIRO
}

\author{
Márcia Zampieri Grohmann \\ Doutora em Engenharia da Produção e Sistemas pela Universidade Federal de Santa Catarina - UFSC \\ Professora do Departamento de Ciências Administrativas da Universidade Federal de Santa Maria - UFSM \\ marciazg@ufsm.br \\ Luciana Flores Battistella \\ Doutora em Engenharia da Produção e Sistemas pela Universidade Federal de Santa Catarina - UFSC \\ Professora do Departamento de Ciências Administrativas da Universidade Federal de Santa Maria - UFSM \\ lutti@ufsm.br \\ Aline Nadalin Velter \\ Mestre em Administração pela Universidade Federal de Santa Maria - UFSM \\ aline.velter@gmail.com \\ Fernanda Casasola \\ Mestranda em Administração da Universidade Federal de Santa Maria - UFSM \\ fernandacasasola@gmail.com
}

\section{RESUMO}

O propósito deste estudo foi o de atestar a aplicabilidade da Escala de Comportamento Ecologicamente Consciente do Consumidor (ECCB), desenvolvida por Roberts (1996), na realidade local. Para tanto, o objetivo principal deste artigo foi o de validar a escala ECCB para o contexto nacional e propor os ajustes necessários. Este estudo foi realizado com 176 alunos do curso de graduação em Administração de uma universidade do RS, caracterizando-se como uma pesquisa descritiva e quantitativa do tipo survey. A adequação da Análise Fatorial Exploratória foi comprovada com o valor do KMO 0,915 e Barlett significativo e seu resultado, após a exclusão de três variáveis com comunalidades inferiores a 0,50 , gerou uma estrutura de três fatores (reciclagem, mudança de comportamento e saúde) que explicam 56,9\% da variância total. Por meio da Análise Fatorial Confirmatória, identificou-se a necessidade de dois procedimentos de melhoria do modelo: a inclusão de uma correlação entre os itens $r 3$ (produtos que possuem grande quantidade de embalagens) e p5 (produtos feitos de recursos naturais escassos); e exclusão do item s3 (pagar mais por produtos sem elementos químicos). Com estas alterações, obteve-se um modelo com índices que comprovam um bom ajuste $\left(\chi^{2}=192,610 ; \chi^{2} / \mathrm{gl}=1,65 ; \mathrm{RMR}=0,082 ; \mathrm{CFI}=0,933\right.$; NFI $=0,873$; NNFI $=0,922 ;$ RMSEA $=0,064)$ e, desta forma, este estudo comprova que após algumas adaptações, a escala apresenta consistência para ser aplicada no contexto nacional.

Palavra-chave: Escala ECCB; Consciência ecológica; Comportamento do consumidor; Validação de escala.

\section{ENVIRONMENTALLY CONSCIOUS CONSUMER BEHAVIOR: ADAPTATION OF ECCB'S SCALE FOR BRAZILIAN CONTEXT}

\section{ABSTRACT}

The purpose of this study was adapted Ecologically Conscious Consumer Behavior (ECCB) Scale, developed by Roberts (1996), for the Brazilian context. Thus, the aim of this paper was to validate the ECCB scale to the national and propose the necessary adjustments. So was made a descriptive survey with 176 university students. The adequacy of exploratory factor analysis was confirmed with the value of the KMO and Barlett 0.915 meaningful and its results, after excluding three variables with communalities less than 0.50 , had a three-factor structure (recycling, behavior change and health) explaining $56.9 \%$ of total variance. Through Confirmatory Factor Analysis, we identified the need for two procedures for improving the model: the inclusion of a correlation between the items $\mathrm{r} 3$ (products that have large amounts of packaging) and $\mathrm{p} 5$ (products made from scarce natural resources), and exclusion Item $\mathrm{s} 3$ (pay more for products with no chemicals). With these changes, we obtained a model with indices show a good fit $\left(\chi^{2}=192.610, \chi^{2} / \mathrm{df}=\right.$ $1.65, \mathrm{RMR}=0.082, \mathrm{CFI}=0.933, \mathrm{NFI}=0.873, \mathrm{NNFI}=0.922, \mathrm{RMSEA}=0.064)$ and thus, the study shows that after some adjustments, this scale showed consistency to be applied in the national context.

Key words: Conscious; Consumer behavior; Ecological; Scale ECCB; Scale validation. 


\section{INTRODUÇÃO}

Nas últimas décadas, têm se observado um aumento progressivo da consciência ambiental do consumidor, por isso, a questão ambiental deixou de ser secundária e tornou-se central para muitas sociedades (Kalafatis, Pollard, East \& Tsogas, 1999). Este aumento da consciência ambiental tem sido impulsionado por uma série de fatores, tais como o aumento da cobertura da mídia, uma maior conscientização dos problemas ambientais, aumento das pressões de grupos ativistas, uma legislação mais rigorosa (nacional e internacional) e o impacto das catástrofes industriais na opinião pública (Butler, 1990; Mcintosh, 1991; Tapon \& Leighton, 1991; Charter, 1992; Wagner, 1997). Consequentemente, os consumidores estão mais preocupados sobre seus hábitos de vida e como eles impactam no meio ambiente (Krause, 1993). Os consumidores também estão mais preocupados com os valores morais, em razão disso, estão mais dispostos a comprar um produto com uma qualidade socioambiental (De Ferran \& Grunert, 2007).

Na literatura existente, vários estudos como os de Kinnear, Taylor e Ahmed, 1974; Buttel, 1979; Schwepker e Cornwell, 1991; Jackson, Olsen, Granzin e Burns, 1993; Shamdasani, Ong Chon-Li e Richmond, 1993; Shrum, McCarty e Lowrey, 1995; Roberts, 1996; Schlegelmilch, Bohlen e Diamantopoulos, 1996; Giannelloni, 1998; Kalafatis, Pollard, East e Tsogas, 1999; Straughan e Roberts, 1999; Follows e Jobber, 2000; Battistella, Velter, Grohmann e Casasola (2012) já atestaram a relação entre a consciência ambiental e sua influência no comportamento de compra do consumidor.

Assim, buscando compreender melhor a relação entre consciência ecológica e comportamento do consumidor, este estudo tem o propósito de identificar a aplicabilidade da Escala de Comportamento Ecologicamente Consciente do Consumidor (ECCB), desenvolvida por Roberts (1996), para o contexto brasileiro. Salienta-se que esta escala já havia sido traduzida por Lages e Neto (2002) e utilizada em outros estudos como o de Pereira, Ayrosa e Fernandez (2004), Jain e Kaur (2004) e Queiroga, Gouveia, Coutinho, Vasconcelos e Jesus (2005). Tanto o estudo de Lages e Neto (2002) quanto o de Queiroga et al (2005) validaram a escala, porém apenas por meio da Análise Fatorial Exploratória e, portanto, não foram utilizados testes estatísticos mais complexos, como a Análise Fatorial Confirmatória (AFC), e também não se buscou mecanismos para a melhoria do modelo.

Dessa forma, as etapas deste estudo foram as seguintes: a) reaplicar a escala de Comportamento Ecologicamente Consciente do Consumidor (ECCB), proposta por Roberts (1996); b) identificar a composição dos fatores da escala; c) utilizar a Análise Fatorial Confirmatória para mensurar o ajuste do modelo global; d) ajustar a escala, visando a melhoria do modelo; e) apresentar a escala final, devidamente validada.

\section{REFERENCIAL TEÓRICO}

A temática Sustentabilidade e suas derivações têm suscitado grande interesse das mais variadas áreas do conhecimento (Velter, Battistella, Grohmann \& Carpes, 2010). O interesse da área gerencial foi intensificado nas décadas de 1960 e 1970 (Kassarjian, 1971; Kinnear, Taylor \& Ahmed, 1974; Webster Jr., 1975; Roberts, 1996; Straughan \& Roberts, 1999; Pereira \& Ayrosa, 2004) e, a partir do final do século XX e, principalmente, na primeira década deste século, ganha atenção entre os mais diversos atores sociais e passa a ser fonte de vantagem competitiva para as organizações e tema de diversos estudos (Queiroga, Gouveia, Coutinho, Vasconcelos \& Jesus, 2005; Peattie, 2001; Robert, Wessells, Donath \& Asche, 2007; Pereira \& Ayrosa, 2004; Cardoso e Cairrão, 2007).

As preocupações com a sustentabilidade e consumo consciente levaram os pesquisadores a buscar parâmetros confiáveis para determinar o grau de envolvimento ambiental de organizações e consumidores. Notadamente, nos estudos realizados com os consumidores buscava-se entender 
valores, atitudes e comportamentos dos consumidores ecologicamente conscientes, bem como descobrir formas de atingir esses consumidores mais efetivamente (Schaefer \& Crane, 2005; Ribeiro \& Teixeira, 2010).

Os estudos sobre consciência ecológica podem ter enfoques distintos, relações com assuntos diversos e, consequentemente, variadas formas de mensuração. A Figura 1 apresenta as principais abordagens no estudo da consciência ecológica: consciência e conhecimento, consciência e relação com qualidade ambiental, comportamento ambiental, reciclagem, e compra consciente.

\section{CONSCIÊNCIAE CONHECIMENTO}

- Arcury et al (1987); Chandler (1972); Diamantopoulos et al (2003); Grunert (1991); Grunert and Kristensen (1994); Lyons e Breakwell (1994); Meffert e Bruhn (1996); Schlegelmilch et al (1996), entre outros.

\section{CONSCIÊNCIAE RELAÇÃO COM QUALIDADE AMBIENTAL}

- Berger e Corbin (1992); Ellen et al (1991); Roberts (1995; 1996); Reberts e Bacon (1997); Weiner e Doescher (1991), entre outros.

\section{COMPORTAMENTO AMBIENTAL}

- Allen e Ferrand (1999); Bratt (1999); Ellen (1994); Ellen et al (1991); Gamba e Oskamp (1994); Gray (1985), entre outros.

\section{RECICLAGEM}

- Aroson (1992); Bratt (1999); Ellen (1994); Geller (1989); McDonald e Volpi (1994); Scott (1999); Oskamp et al (1991), entre outros.

\section{COMPRA CONSCIENTE}

- Balderjahn (1988); Bratt (1999); Coddington (1993); Davis (1993); Ottman (1993), entre outros.

Figura 1: Abordagens nos estudos sobre consciência ambiental.

Fonte: Elaborado pelos autores.

Este estudo toma por base o estudo de Roberts e, portanto, pode ser classificado como tendo foco na relação entre consciência e relação com qualidade ambiental. Porém, seu foco principal é na compra consciente, ou seja, busca compreender como a consciência ecológica afeta os hábitos de consumo das pessoas e, em consequência, seus futuros comportamentos de compra.

Pesquisadores como Maloney, Ward e Braucht (1975) desenvolveram uma escala visando estudar o comprometimento verbal e real, grau de envolvimento afetivo ambiental e conhecimento ecológico. Stone, Barnes e Montgomery (1995), desenvolveram a Ecoscale, com 31 itens estudados em sete dimensões (opiniões e crenças do consumidor sobre a relação humana com o meio ambiente; conscientização sobre impactos ambientais do consumo; disposição de agir para proteger o meio ambiente; atitudes relativas a remediar os problemas ambientais, ações de consumo ecologicamente responsável; habilidade de agir em defesa do meio ambiente; e conhecimento a respeito das questões ecológicas). Posteriormente, Fraj e Martinez (2006, 2007) estudaram personalidade e atitudes ambientais partindo de uma versão reduzida da escala anterior. 
Porém, conforme argumentam Queiroga, et al (2005), entre os estudos que mais se destacam na busca de um modelo de mensuração da consciência ecológica, e de sua relação com os hábitos dos consumidores, está a Escala de Comportamento Consciente do Consumidor (ECCB), desenvolvida por Roberts (1996).

O autor propôs uma escala a partir de uma revisão da literatura existente e de estudos realizados anteriormente que tinham como propósito verificar as variáveis que afetavam o comportamento socialmente responsável durante o processo de decisão de compra dos consumidores. A pesquisa realizada com 582 consumidores adultos nos Estados Unidos, revelou o perfil dos consumidores ecologicamente conscientes da década de 1990 e trouxe implicações para as estratégias de comunicação de marketing e para a academia. Neste estudo, assume-se que o consumidor ecologicamente consciente é aquele que compra produtos que considera ter impacto favorável ao ambiente (Roberts, 1996).

O modelo original da Escala ECCB apresentou como resultado uma estrutura com dois fatores - primeiro com 22 itens, explicando 45,9\% da variância e um segundo fator com 8 itens (em comum mencionavam poupar dinheiro), explicando 6,3\% da variância. $\mathrm{O}$ autor excluiu o segundo fator e ficou com uma estrutura unifatorial de 22 itens e com um alpha de Cronbach de 0,96 (Roberts, 1996).

Observa-se que os itens que formavam o fator 2 no estudo de Roberts (1996) são aqueles que no estudo de Lages e Neto (2002) formaram os fatores hábitos domésticos e uso de energia. Assim, seguindo os mesmos procedimentos adotados por Roberts (1996), opta-se, neste estudo, pela exclusão destas variáveis. Dessa forma, a escala que será testada é composta pelos 22 itens não excluídos por Roberts (o que no estudo de Lages e Neto representam os itens dos fatores: produto, reciclagem, alimentação e saúde, ações de mudança).

Outro estudo nacional que trabalhou com o mesmo modelo foi o de Queiroga, et al (2005), em que se buscou a adaptação da escala de Roberts (1996) que os autores chamaram de Escala de Comportamentos Socialmente Responsáveis do Consumidor (ECSRC). A amostra, assim como a deste estudo, foi composta por estudantes universitários e, por meio de uma análise fatorial dos eixos principais, encontrou-se um modelo com três fatores.

Já em 1999, Straughan e Roberts (1999) reaplicam e estendem a pesquisa conduzida por Roberts, em 1996, e que teve como objetivo principal verificar a existência de correlação entre as variáveis demográficas e psicográficas com a consciência ecológica do consumidor. Assim, Straughan e Roberts (1999), com o propósito de examinar mais detalhadamente o papel das características psicográficas e demográficas sobre o comportamento ecologicamente consciente do consumidor, adicionaram ao estudo de Roberts (1996) outras variáveis que também poderiam caracterizar o perfil do "consumidor verde", em combinação com o constructo anteriormente desenvolvido. A escala desenvolvida e adaptada por Straughan \& Roberts (1999) possui 30 variáveis operacionalizadas numa escala de 5 (cinco) pontos Tipo Likert com valores "Always True" e "Never True". O estudo, realizado com uma amostra de 235 estudantes, encontrou consistência com o estudo anterior realizado por Roberts (1996) e revelou que as características psicográficas mostram-se mais efetivas na explicação do comportamento do consumidor ecologicamente consciente.

Devido o propósito deste estudo, de identificar a aplicabilidade da Escala de Comportamento Ecologicamente Consciente do Consumidor (ECCB), para o contexto brasileiro, optou-se pela utilização da escala de Roberts (1996) para identificar o comportamento do consumidor brasileiro em relação às questões ecológicas. 


\section{MÉTODO DA PESQUISA}

Com o objetivo de validar, no Brasil, a Escala de Comportamento Ecologicamente Consciente do Consumidor (ECCB), foi realizada uma pesquisa descritiva e quantitativa com uma amostra inicial de 176 estudantes universitários. A escolha por este tipo de amostra (universitários) deveu-se ao argumento de Straughan e Roberts (1999) de que é muito importante mensurar o futuro do consumo consciente e, para tanto, nada melhor do que focar estes estudos nos jovens que serão formadores de opinião.

O nível de comportamento ecologicamente consciente foi medido por meio da escala desenvolvida por Roberts (1996), conhecida como ECCB. Assim, a coleta de dados foi realizada por meio de questionários compostos exclusivamente de questões fechadas que utilizam escala Likert de cinco pontos ( $1=$ discordo totalmente; $5=$ concordo totalmente), sendo 22 questões referentes a escala ECBB e cinco questões que relacionavam-se com características sociodemográficas dos respondentes. Destaca-se, contudo, que um item - evito utilizar meu carro para não poluir o ambiente - posteriormente foi excluído do modelo, pois, pelo fato da amostra ser de universitários, essa questão obteve muitas respostas em branco.

Seguindo o procedimento proposto por Malhotra (2001), a escala foi traduzida para o português por meio da técnica de tradução reversa e realizou-se um pré-teste (com 10 universitários) para a identificação de possíveis problemas. Esse procedimento demonstrou a necessidade de adaptação de alguns termos para uma linguagem mais informal. Destacam-se que os itens traduzidos foram confrontando com os do estudo de Lages e Neto (2002), autores que também traduziram a escala ECCB, e observou-se pouca alteração no formato das questões.

Para que a validação de um modelo ocorra, por meio da Análise Fatorial Confirmatória, antes é necessária uma análise preliminar dos dados, por meio de estatísticas descritivas, análise fatorial exploratória e avaliação da confiabilidade e validade das escalas (Hair, Tatham, Anderson \& Black, 2005). Os dados foram analisados por meio de estatísticas descritivas, Análise Fatorial Exploratória (AFE), realizada com o software PASW Statistics 17, a Análise Fatorial Confirmatória (AFC) e refinamento do modelo, realizadas com o software AMOS 16.0.

\section{RESULTADOS}

A amostra desta pesquisa foi composta por 176 estudantes universitários, sendo que, quanto ao gênero, 89 eram mulheres $(50,3 \%)$ e 88 homens $(49,7 \%)$. Em relação à idade, a amplitude encontrada foi grande, variando de 17 a 45 anos, sendo que a moda foi de 19 anos e a média de 21,43 anos (com desvio-padrão de 4,04336).

Grande parte destes estudantes ainda mora com os pais e são solteiros $(54,1 \%)$, outra parcela considerável $(36,9 \%)$ mora com amigos ou sozinho e apenas $8,2 \%$ dos entrevistados moram com cônjuge ou companheiro, sendo que 5,7\% não têm filhos e 2,5\% têm filhos. Além disto, para concluir o perfil da amostra, salienta-se que 51,6\% dos entrevistados apenas estudam e que 47,8\% estudam e trabalham. Por fim, para a identificação da classe econômica, foi utilizado o Critério de Classificação Econômica Brasil de 2008, e os resultados indicaram que a maioria dos universitários entrevistados enquadra-se como classe A2 $(26,7 \%)$ e Classe B1 (26,1\%). Na sequência aparecem as classes B2 (23,3\%), C1 (12,5\%), A1 (9\%) e C2 (2,3\%).

Após um breve comentário sobre a amostra do estudo, são apresentados os dados sobre a validação da Escala de Consciência Ecológica (Roberts, 1996) e, para tanto, seguindo os procedimentos propostos por Hair et al. (2005), os dados foram submetidos a uma análise fatorial exploratória (AFE) e a uma análise fatorial confirmatória (AFC).

A AFE foi realizada com o objetivo de examinar a estrutura dos dados e agrupá-los em categorias (os fatores). Já na AFC, o objetivo principal foi o de verificar a validade e a confiabilidade do instrumento resultante da AFE. 


\subsection{Estrutura dos dados - análise fatorial exploratória}

Nesta etapa, foi utilizada análise fatorial com rotação Varimax, que maximiza o peso de cada variável dentro de cada fator (Malhotra, 2001), e para a extração dos fatores utilizou-se o critério de autovalor maior que 1 (um).

Para mensurar a adequação da aplicação da análise fatorial foram utilizados os dois índices mais comuns: esfericidade de Bartlett e Kaiser-Meyer-Olkin (KMO). O valor do KMO encontrado na pesquisa foi de 0,915, altamente satisfatório, muito próximo de um e bem superior aos 0,5 , considerados como limite mínimo satisfatório. Assim, comprova-se que a correlação entre cada par de variáveis pode ser explicada pelas outras variáveis que formam o construto. $\mathrm{O}$ valor do teste de esfericidade de Barlett foi de 1266,695, com nível de significância de 0,000, indicando que a correlação entre as variáveis é significativa, a ponto de apenas alguns fatores poderem representar grande parte da variabilidade dos dados.

Tabela 1: Análise fatorial exploratória.

\begin{tabular}{|c|c|c|c|}
\hline \multirow{2}{*}{ VARIÁVEL } & \multicolumn{3}{|c|}{ Fator } \\
\hline & reciclagem & mudança & saúde \\
\hline r1 - Produtos feitos de papel reciclado & 0,792 & 0,058 & 0,110 \\
\hline r4 - Produtos que podem ser reciclados & 0,780 & 0,125 & 0,281 \\
\hline r2 - Produtos feitos com material reciclado & 0,655 & 0,266 & 0,152 \\
\hline r5 - Produtos com embalagens que não são biodegradáveis. & 0,617 & 0,421 & 0,231 \\
\hline r3 - Produtos que possuem grande quantidade de embalagens & 0,562 & 0,537 & 0,091 \\
\hline p6 - Produtos que causam menor poluição & 0,556 & 0,235 & 0,334 \\
\hline p5 - Produtos feitos de recursos naturais escassos & 0,525 & 0,507 & 0,169 \\
\hline p2 - Produtos que prejudicam o meio ambiente & 0,462 & 0,314 & 0,453 \\
\hline $\begin{array}{l}\text { p3 - Produto que é menos prejudicial às pessoas e ao meio } \\
\text { ambiente }\end{array}$ & 0,112 & 0,781 & 0,018 \\
\hline $\begin{array}{l}\text { p4 - Convencer a não comprar produtos que prejudicam o } \\
\text { ambiente }\end{array}$ & 0,209 & 0,748 & 0,057 \\
\hline m3 - Comprar produto que causa danos & 0,183 & 0,660 & 0,379 \\
\hline m2 - Comprar produtos que podem causar a extinção & 0,205 & 0,581 & 0,383 \\
\hline m1 - Deixar de usar produtos por razões ecológicas & 0,378 & 0,496 & 0,374 \\
\hline $\begin{array}{l}\text { p1 - Comprar produtos de empresas que prejudicam o meio } \\
\text { ambiente }\end{array}$ & 0,244 & 0,450 & 0,446 \\
\hline s1 - Comprar produtos orgânicos & 0,196 & 0,079 & 0,785 \\
\hline s3 - Pagar mais por produtos sem elementos químicos & 0,085 & 0,061 & 0,682 \\
\hline s2 - Preferir alimentos sem agrotóxicos & 0,211 & 0,188 & 0,669 \\
\hline s4 - Preocupações ambientais interferem na decisão de compra & 0,473 & 0,270 & 0,531 \\
\hline Variância explicada & $21,142 \%$ & $19,317 \%$ & $16,475 \%$ \\
\hline Alpha de Cronbach & 0,876 & 0,822 & 0,730 \\
\hline
\end{tabular}

Quanto às comunalidades, três itens apresentaram valores inferiores a 0,50 e, dessa forma, optou-se por extraí-los do modelo, utilizando-se o critério de comunalidade inferior a 0,50. Os itens

Revista de Gestão Social e Ambiental - RGSA, São Paulo, v. 6, n. 1, p. 102-116, jan./abril 2012. 
excluídos foram: utilizo centros de reciclagem, utilizo detergentes que causam pouco impacto ao ambiente; não compro produtos em embalagens do tipo aerosol.

As cargas fatoriais das variáveis que formam o modelo foram quase todas superiores a 0,60 e apenas duas variáveis ( $\mathrm{p} 2$ - comprar produtos que prejudicam o meio ambiente - e p1 - comprar produtos de empresas que prejudicam o meio ambiente) obtiveram cargas inferiores a 0,50 , porém muito próximas (acima de 0,45 ).

A estrutura fatorial encontrada, Tabela 1, foi de três fatores denominados de: reciclagem, mudança de comportamento e saúde. As cargas fatoriais foram elevadas, como será detalhado na análise fatorial confirmatória, e a variância total explicada pelos três fatores foi de 56,934\%. O fator reciclagem contribuiu para $21,142 \%$ da variância; o fator mudança de comportamento para 19,317\% e o fator saúde para $16,475 \%$.

Por fim, para avaliar a confiabilidade da escala adotou-se o cálculo do coeficiente Alpha de Cronbach. Os resultados para cada um dos fatores foram: reciclagem $=0,876$; mudança de comportamento $=0,822$; saúde $=0,730$. Estes índices são considerados bons, pois segundo Malhotra (2001) aceitam-se resultados acima de 0,60. E o valor do alpha de Cronbach para todo o instrumento foi de 0,918, ou seja, melhor ainda que os valores encontrados nos fatores. Assim, concluí-se que o instrumento possui uma consistência interna satisfatória.

Após esta etapa, é necessário salientar que a estrutura fatorial encontrada não foi a mesma do estudo de Lages e Neto (2002), pois naquela ocasião os autores identificaram quatro fatores. No entanto, os dados apresentaram uma estrutura similar ao estudo de Queiroga et al (2005), tendo uma estrutura de três fatores, mas os itens que formaram cada fator, sofreram certa variação. Como é possível observar na Tabela 2, neste estudo os itens que originalmente deveriam medir o construto Produto, mesclaram-se com os itens do construto Mudança e Reciclagem. Dessa forma, o único construto que permaneceu igual ao do estudo de Lages e Neto (2002) foi Saúde. No que se refere ao modelo original (Roberts, 1996), a estrutura encontrada não foi unifatorial e, além disso, o número de itens foi alterado de 22 para 18.

Tabela 2: Índices comparativos das pesquisas.

\begin{tabular}{|l|c|c|l|l|}
\hline Estudo & $\mathbf{N}^{\mathbf{0}}$ fatores & $\mathbf{N}^{\mathbf{0}}$ itens & \multicolumn{1}{|c|}{$\begin{array}{c}\text { Variância } \\
\text { Explicada }\end{array}$} & \multicolumn{1}{c|}{ Alfas } \\
\hline Roberts (1996) & 01 & 22 & $52,2 \%$ & Geral - 0,96 \\
\hline Lages e Neto (2002) & 04 & 18 & $46,3 \% *$ & $\begin{array}{l}\text { Produto - 0,80 } \\
\text { Reciclagem - 0,79 } \\
\text { Alimentação saúde - 0,78 } \\
\text { Ação de mudança - 0,52 } \\
\text { Geral - 0,90 }\end{array}$ \\
\hline $\begin{array}{l}\text { Queiroga et al } \\
\text { (2005) }\end{array}$ & 03 & 15 & $41,1 \%$ & $\begin{array}{l}\text { Preocupação ambiental - 0,90 } \\
\text { Reciclagem - 0,75 } \\
\text { Preocupação social - 0,70 } \\
\text { Geral - não informado }\end{array}$ \\
\hline Este estudo & 03 & 18 & $56,93 \%$ & $\begin{array}{l}\text { Reciclagem - 0,88 } \\
\text { Mudança - 0,82 } \\
\text { Saúde - 0,73 } \\
\text { Geral - 0,92 }\end{array}$ \\
\hline
\end{tabular}

* percentual equivalente aos quatro fatores utilizados (dos seis constantes no modelo original).

Sobre a confiabilidade da escala (Tabela 2), observa-se que neste estudo o valor do Alpha de Cronbach foi superior (de 0,92 para 0,90) ao encontrado por Lages e Neto (2002), já em comparação com Roberts (1996), o alpha foi um pouco inferior (de 0,96 para 0,92) e, no estudo de 
Queiroga, este valor não foi informado, porém, os alphas por fatores obtiveram resultados semelhantes. Por fim, analisando-se o total da variância explicada, observa-se que resultados variaram entre $41 \%$ a $57 \%$, e que o percentual deste estudo foi o mais elevado, porém nenhum dos modelos atingiu o limite indicado de $60 \%$ de explicação.

Dessa forma, o resultado da AFE resultou no Modelo Inicial de Comportamento Ecologicamente Consciente do Consumidor (Figura 2), formado por três construtos: saúde, reciclagem e mudança. O construto reciclagem está composto de oito itens (cinco que no trabalho de Lages e Neto eram do construto Reciclagem e três do construto Produto); o construto Mudança de Atitude é formado por seis itens (três que no trabalho de Lages e Neto eram do construto Mudança e três do construto Produto); e o construto Saúde, que permaneceu igual ao trabalho de Lages e Neto (2002), conta com quatro itens.

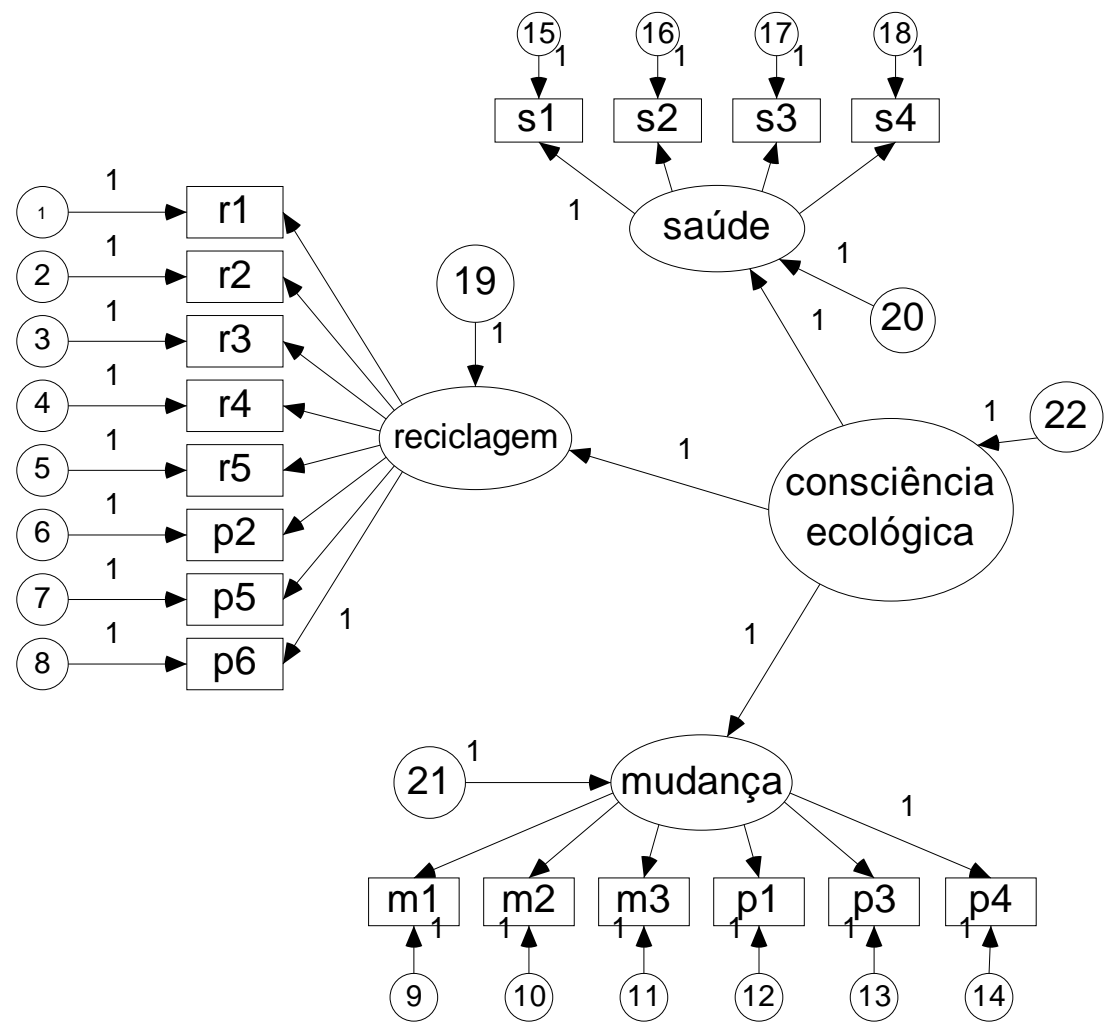

Figura 2: Modelo estrutural proposto.

Fonte: Elaborado pelos autores.

\subsection{Validade da escala - análise fatorial confirmatória}

Para a validação do modelo inicial, foi realizada a Análise Fatorial Confirmatória (AFC) com os 176 questionários, todos respondidos de forma completa, ou seja, não existindo dados omissos. Há divergências sobre o tamanho ideal de uma amostra para a realização da AFC, porém, seguindo o argumento de Hair et al. (2005), julgou-se o tamanho adequado, pois o modelo proposto enquadra-se nas três condições para a utilização de amostras entre 100 a 150 questionários: modelos contendo até cinco construtos, cada um com, no mínimo, três itens de mensuração e com comunalidades elevadas.

Antes de proceder a AFC, foram analisados os casos extremos (outliers) e a normalidade das variáveis. Por meio do teste realizado pelo próprio software Amos - Distância de Mahalanobis -, identificou-se que não havia casos extremos, pois nenhum item distanciou-se mais de 1,5 desvios- 
quartílicos do quartil superior ou do quartil inferior. Porém, no que diz respeito à normalidade, os gráficos Q-Q e P-P demonstraram que boa parte dos dados apresentavam distribuição normal, o que segundo Hair et al (2005) é o pressuposto mais importante da AFC. Mas, para a comprovação da normalidade foi realizado o teste Kolmogorov-Smirnov (KS) que demonstrou que algumas distribuições não seguiam a normalidade.

Apesar da falta de normalidade em alguns itens, optou-se por realizar a AFC por meio da máxima verossimilhança. Porém, antes de se apresentar os índices de ajuste do modelo é importante, segundo Garver e Mentzer (1999), analisar estatisticamente algumas propriedades que devem ser satisfeitas para que a validade do construto seja alcançada. Tais propriedades são: unidimensionalidade, validade convergente, validade discriminante dos construtos do modelo.

A unidimensionalidade representa o grau em que os itens representam apenas uma variável latente e para sua identificação foi utilizado o critério dos resíduos padronizados. Resíduos padronizados menores que 2,58 $(\mathrm{p}<0,05)$ atestam a unidimensionalidade dos itens e, conforme pode ser observado na Tabela 3, todos os valores de resíduos do modelo foram bem inferiores a este limite (os maiores resíduos foram os dos itens s4 (preocupações ambientais interferem na decisão de compra) com 0,62 e r5 (produtos com embalagens que não são biodegradáveis) com valor de 0,58). Assim, a unidimensionalidade do modelo foi comprovada.

Tabela 3: Cargas fatoriais e erros padronizados.

\begin{tabular}{|c|c|c|c|c|}
\hline Construto & Item & Carga & t-value & Erro \\
\hline Reciclagem & R1 & 0,614 & 8,218 & 0,38 \\
& R2 & 0,642 & 8,159 & 0,41 \\
& R3 & 0,709 & 7,818 & 0,50 \\
& R4 & 0,732 & 7,676 & 0,54 \\
& R5 & 0,761 & 7,513 & 0,58 \\
& P2 & 0,670 & 8,018 & 0,45 \\
& P5 & 0,708 & 7,893 & 0,50 \\
\hline \multirow{6}{*}{ Mudança } & P6 & 0,657 & 8,103 & 0,43 \\
& M1 & 0,685 & 7,592 & 0,47 \\
& M2 & 0,671 & 7,706 & 0,45 \\
& M3 & 0,715 & 7,355 & 0,51 \\
& P1 & 0,644 & 7,822 & 0,42 \\
& P3 & 0,573 & 8,095 & 0,33 \\
& P4 & 0,618 & 7,928 & 0,38 \\
\hline \multirow{2}{*}{ Saúde } & S1 & 0,621 & 7,620 & 0,39 \\
& S2 & 0,632 & 7,587 & 0,40 \\
& S3 & 0,471 & 8,302 & 0,22 \\
& S4 & 0,786 & 6,236 & 0,62 \\
\hline
\end{tabular}

Para a identificação da validade convergente, ou seja, verificar se indicadores que supõem medir o mesmo fenômeno estão correlacionados (Hair et al., 2005), foram realizados dois procedimentos. Em primeiro lugar, por meio das análises de correlações entre os fatores (Tabela 3) identificou-se existência de correlações significativas entre todos os pares de fatores. Num segundo momento, utilizou-se o critério do t-value, ou seja, existe validade convergente quando as cargas fatoriais apresentam-se significativas ao nível de 0,01 ( $t$-value $\geq 2,33$ ). A Tabela 3 apresenta essas informações e demonstra que todos os valores foram bem superiores aos 2,33 (menor valor s4 - 
preocupações ambientais interferem na decisão de compra - com valor de 6,263), o que comprova a validade convergente de todos os itens.

Por fim, para avaliação da validade discriminante, ou seja, extensão pela qual a escala não se correlaciona com medidas de outros construtos, foi utilizada a comparação entre a variância extraída da dimensão e suas variâncias compartilhadas. Como todas as dimensões apresentaram variância extraída maiores do que as variâncias compartilhadas foi comprovado que a validade discriminante do modelo é positiva.

Além dessas análises, observa-se que não foram encontradas variâncias-erro negativas, coeficientes padronizados com valores acima de 1,00 e erros padronizados muito grandes, o que demonstra que o modelo não apresenta estimativas discrepantes.

Para o ajuste do modelo, procedeu-se a análise dos índices comumente utilizados: estatística qui-quadrado $\left(\chi^{2}\right)$, qui-quadrado/graus de liberdade $\left(\chi^{2} / \mathrm{gl}\right)$ root mean square residual (RMR), root mean square error of approximation (RMSEA), comparative fit index (CFI), normed fit index (NFI) e non normed fit index (NNFI).

O modelo inicial (Figura 2) apresentou índices satisfatórios de ajuste. Os valores encontrados foram de: $\chi^{2}=239,060 ; \chi^{2} / \mathrm{gl}=1,78 ; \mathrm{RMR}=0,088$; CFI $=0,910$; NFI $=0,820$; NNFI $=0,898$; RMSEA $=0,071$. Os valores recomendados como ideais são superiores a 0,90 para CFI, NFI e NNFI; inferiores a 0,10 para RMR e RMSEA e máximo de 5,00 para $\chi^{2} / \mathrm{gl}$. Assim, observa-se que apenas o valor do NFI não foi satisfatório.

Analisando-se as sugestões de melhoria do modelo, propostas pelos outputs do software Amos, foram adotados dois procedimentos: o primeiro, a inclusão de uma correlação entre itens; e o segundo, a exclusão de um item com carga fatorial inferior a 0,50 .

A correlação acrescentada foi entre os itens $r 3$ (produtos que possuem grande quantidade de embalagens) e p5 (produtos feitos de recursos naturais escassos), o que denota a preocupação de preservação de recursos naturais escassos que seriam prontamente descartados ante sua utilização em embalagens com excessivo uso de recursos.

Já o item excluído foi o s3 (pagar mais por produtos sem elementos químicos), de certa forma, isso pode ser atribuído ao fato que os consumidores não consideram ético que as empresas cobrem mais por produtos sem elementos químicos, devendo buscar competitividade com os demais produtos.

O novo modelo, denominado de Modelo Final, apresentou melhora em todos os itens. Dessa forma, a avaliação do ajuste geral do modelo foi de: $\chi^{2}=192,610 ; \chi^{2} / \mathrm{gl}=1,65 ; \mathrm{RMR}=0,082 ; \mathrm{CFI}=$ 0,933 ; NFI $=0,873 ;$ NNFI $=0,922$; RMSEA $=0,064$. Assim, apenas o NFI continuou fora dos limites ideais, apesar de apresentar uma considerável melhora. Destaca-se que o NFI, segundo Hair et al. (2005, p.571), é uma medida de "[...]proporção da diferença no valor do $\chi^{2}$ para o modelo ajustado e um modelo nulo dividido pelo valor $\chi^{2}$ para o modelo nulo[...]", ou seja, aponta a proporção em que o ajuste do modelo proposto é melhor do que o do modelo nulo.

Pela avaliação da matriz de resíduos normalizados, observou-se que o menor valor encontrado foi de $-2,336$ e o maior valor, de 1,902. Isso demonstra que nenhum valor ficou fora do intervalo 2,58 a 2,58 (valores para significância de 0,05), ou seja, o modelo teve $0 \%$ de dados fora dos limites, sendo que se aceita um valor de até $5 \%$, fato que corrobora com o bom ajuste do modelo.

Dessa forma, após os testes estatísticos (AFE e AFC) e as alterações realizadas para a melhoria do modelo, o instrumento final, ou seja, a escala ECCB adaptada ao contexto nacional é apresentada na Figura 3. Salienta-se que a mesma deve ser aplicada por meio de uma escala do tipo Likert de cinco pontos, na qual 1 equivale a "concordo totalmente" e 5 equivale a "discordo totalmente". 


\section{FATOR RECICLAGEM}

01 Eu procuro comprar produtos feitos de papel

02 Eu tento comprar apenas produtos que podem ser reciclados

03 Sempre que possível, eu compro produtos feitos com material reciclado

04 Eu evito comprar produtos com embalagens que não são biodegradáveis

05 Eu procuro não comprar produtos que possuem grande quantidade de embalagens

06 Quando possível, eu sempre escolho produtos que causam menor poluição

$07 \mathrm{Eu}$ sempre faço um esforço para reduzir o uso de produtos feitos de recursos naturais escassos

08 Eu não compro produtos para minha casa que prejudicam o meio-ambiente

\section{FATOR MUDANÇA DE HÁBITO}

09 Quando eu tenho que escolher entre dois produtos iguais, eu sempre escolho o que é menos prejudicial às outras pessoas e ao meio-ambiente

10 Eu já convenci amigos ou parentes a não comprar produtos que prejudicam o meioambiente

11 Quando eu conheço os possíveis danos que um produto pode causar ao meio ambiente, eu não compro este produto

12 Eu não compro produtos e alimentos que podem causar a extinção de algumas espécies animais e vegetais

13 Eu já troquei ou deixei de usar produtos por razões ecológicas

$14 \mathrm{Eu}$ não compro produtos fabricados ou vendidos por empresas que prejudicam ou desrespeitam o meio-ambiente

\section{FATOR SAÚDE}

15 Eu compro produtos orgânicos porque são mais saudáveis

16 Eu prefiro produtos e alimentos sem agrotóxicos porque eles respeitam o meio ambiente

17 Quando eu compro produtos e alimentos as preocupações com o meio ambiente interferem na minha decisão de compra

Figura 3: Escala ECCB adaptada ao contexto brasileiro.

Fonte: Elaborado pelos autores.

\section{CONSIDERAÇÕES FINAIS}

O tema escolhido para este estudo foi o de relacionar comportamento do consumidor com consciência socioambiental e, num primeiro momento, constatou-se a relevância do assunto, pois foram encontrados muitos estudos recentes com esta temática. Dessa forma, concorda-se com a afirmação de Roberts (1996) de que a preocupação ambiental é um tema que preocupa as gerações atuais e que está frequentemente relacionado com os comportamentos dos consumidores.

Porém, é importante destacar que a maior parte das pesquisas que relaciona os dois aspectos identifica que a consciência ambiental é fracamente correlacionada com o comportamento de compra (Queiroga, et al, 2005). Porém, estudos como os de Garcia, Silva, Pereira, Rossi e Bazanini (2008) e Battistella, Velter, Grohmann e Costa (2012) identificaram uma forte relação (mais de $70 \%$ ) entre importância atribuída pelos consumidores ao comportamento ambientalmente 
responsável e a propensão do consumidor a recompensar o comportamento com responsabilidade ambiental das empresas.

O propósito deste estudo foi de adaptar, para o contexto brasileiro, a Escala de Comportamento Ecologicamente Consciente do Consumidor (ECCB). E, por meio do referenciado teórico, comprovou esta necessidades, pois alguns dos estudos nacionais que utilizaram esta escala (Lages \& Neto, 2002; Queiroga, et al, 2005), encontraram resultados de validação bem distintos, conforme apontou a Tabela 2.

Os resultados encontrados na primeira parte das análises desta pesquisa (Análise Fatorial Exploratória) identificaram uma estrutura de 18 variáveis, agrupadas em três fatores: reciclagem, mudança de comportamento e saúde. Este modelo apresentou uma boa confiabilidade, pois o alpha de Cronbach foi de 0,92 e explica 56,93\% da variância total encontrada nas respostas.

Na segunda etapa da análise dos dados, com a Análise Fatorial Confirmatória, comprovou-se a unidimensionalidade do modelo, pois todos os resíduos padronizados apresentaram-se menores que 2,58, a validade convergente de todos os itens e atestou-se que a validade discriminante do modelo é positiva.

Em relação ao ajuste final do modelo, os índices encontrados foram: $\chi^{2}=192,610 ; \chi^{2} / \mathrm{gl}=$ 1,$65 ; \mathrm{RMR}=0,082 ; \mathrm{CFI}=0,933 ; \mathrm{NFI}=0,873 ; \mathrm{NNFI}=0,922 ; \mathrm{RMSEA}=0,064$. Todos são considerados bons e comprovam o bom ajuste do modelo de mensuração, ou seja, atestam a consistência do instrumento de coleta proposto.

Em suma, o processo de adaptação da escala ECCB para o contexto nacional exigiu as seguintes alterações no modelo proposto por Roberts (1996):

i) mudança de uma estrutura unifatorial para uma estrutura de três fatores;

ii) três itens, com comunaliades inferiores a 0,50 foram excluídos da Análise Fatorial Exploratória (utilizo centros de reciclagem, utilizo detergentes que causam pouco impacto ao ambiente; não compro produtos em embalagens do tipo aerosol);

iii) inclusão da uma correlação entre os itens $r 3$ (produtos que possuem grande quantidade de embalagens) e p5 (produtos feitos de recursos naturais escassos);

iv) exclusão para a melhoria do modelo, na Análise Fatorial Confirmatória, do item s3 (pagar mais por produtos sem elementos químicos).

Entre as limitações enfrentadas por este estudo, apontam-se o perfil jovem dos respondentes e por serem estudantes de Administração, compondo uma amostra homogênea. Sugere-se que futuros estudos ampliem para uma amostra mais probabilística de composição heterogênea ou que se amplie o número de respondentes.

Porém, apesar desta ser uma contribuição inicial ao tema, considera-se que os resultados encontrados foram significativos e que o instrumento proposto apresenta consistência. Dessa forma, espera-se que a escala proposta seja utilizada em novos estudos.

\section{REFERÊNCIAS}

Battistella, L. F., Velter, A. N., Grohmann, M. Z., \& Costa, V. F. (2012). Avaliação da importância percebida e da recompensa atribuída pelos consumidores a empresas com responsabilidade sociombiental. Revista Alcance, 19, 35-51.

Battistella, L. F., Velter, A. N., Grohmann, M. Z., \& Casasola, F. (2012). Aplicação da Escala-NEP para a mensuração da consciência ecológica de professores universitários: perfil e Implicações para Estudos Futuro. Desenvolvimento em Questão, 10, 219-250.

Butler, D. (1990) A deeper shade of green. Management Today, 158-67, June. 
Cardoso, A. J. M., \& Cairrão, A. M. C. (2007) Os jovens universitários e o consumo sustentável. A sua influência na compra de produtos ecológicos. Revista da Faculdade de Ciência e Tecnologia, 4, 124-135.

Charter, M. (1992) Greener marketing: a responsible approach to business. Greenleaf, Sheffield.

De Ferran F., \& Grunert K. (2007) French fair trade coffee buyers' purchasing motives. Food Quality and Preferences, 18, 218-229.

Follows, S. B., \& Jobber D. (2000) Environmentally responsible purchase behavior: a test of a consumer model. European Journal of Marketing, 34(5/6), 723-746.

Fraj, E., \& Martinez, E. (2006) Influence of personality in ecological consumer behaviour. Journal of Consumer Behavior, 5, 167-181.

Fraj, E. (2007) Ecological consumer behaviour: an empirical analysis. International Journal of Consumer Studies, 31, 26-33.

Garcia, M. N., Silva, D., Pereira, R. S., Rossi, G. B., \& Bazanini, R. (2008) Percepção sobre a recompensa do consumidor ao comportamento empresarial socioambientalmente responsável. Anais do Encontro Nacional da Associação Nacional de Pós-Graduação e Pesquisa em Administração, v.32, Rio de Janeiro.

Garver, M. S., \& Mentzer, J. T. (1999) Logistics research methods: employing structural equation modeling to test for construct validity. Journal of Business Logistics, 20(1), 33-57.

Giannelloni, J-L. (1998) Les comportements liés à lá protection de l'environnment et leurs déterminants: un état des recherches en marketing. Recherche et Apllications en Marketing, 13( 2), 49-72.

Hair Jr., J. F., Tatham, R. L.; Anderson, R. E., \& Black, W. (2005). Análise multivariada de dados. Porto Alegre: Bookman.

Jackson, A. L., Olsen, J. E., Granzin, K. L., \& Burns, A. C. (1993) An investigation of determinants of recycling consumer behavior. Advances in Consumer Research, 20, 481-487.

Jain, S., \& Kaur, G. (2004) Green marketing: an attitudinal and behavioural analysis of Indian consumers. Global Bussiness Review, 5(4), 187- 205.

Kalafatis, S. P., Pollard, M., East, R., \& Tsogas, M. H. (1999) Green marketing and Azjen's theory of planned behaviour: a cross-market examination. The Journal of Consumer Marketing, 16(5), $441-460$.

Kassarjian, H. H. (1971) Incorporating ecology into marketing strategy: the case of air pollution. Journal of Marketing, 35, 61-65.

Kinnear, T. C., Taylor; J. R., \& Ahmed, S. A. (1974) Ecologically concerned consumers: who are they? Journal of Marketing, 38(2), 20-24. 
Krause, D. (1993) Environmental consciousness: an empirical study. Journal of Environment and Behavior, 25(1), 126-42.

Lages, N., \& Neto, A. V. (2002) Mensurando a consciência ecológica do consumidor: um estudo realizado na cidade de Porto Alegre. Anais do Encontro da Associação Nacional de Pós-Graduação e Pesquisa em Administração: v..26. Salvador.

Malhotra, N. K. (2001) Marketing research: an applied orientation. Upper Saddle River: Prentice Hall.

Maloney, M. P., Ward, M. P., \& Braught, G. N. (1975) Psychology in action: a revised scale for the measurement of ecological attitudes and knowledge. American Psychologist, 30, 787-790.

Mcintosh, A. (1991) The impact of environmental issues on marketing and politics in the 1990s. Journal of the Market Research Society, 33(3), 205-217.

Peattie, K. (2001) Golden goose or wild goose? The hunt for the green consumer. Business Strategy and the Environment, 10(4), 187-199.

Pereira, S. J. N., \& Ayrosa, E. A. T. (2004) Atitudes relativas a marcas e argumentos ecológicos: um estudo experimental. Revista Gestão.org, 2(2), 134-145.

Queiroga, F., Gouveia, V. V., Coutinho, M. P., Vasconcelos, T. C., \& Jesus, G. R. (2005) Escala de comportamentos socialmente responsáveis do consumidor: estudo preliminar de evidência de validade. Psicologia e Estudo, 10(1), 143-149.

Ribeiro, J. A., \& Veiga, R. T. (2010) Proposição de uma escala de consumo sustentável. Anais do Encontro de Marketing, v. 4, Florianópolis.

Robert, J., Wessells, C., Donath, H., \& Asche, F. (2007) Measuring consumer preferences for ecolabeled seafood: an international comparison. Journal of Agricultural and Resource Economics, 26(1), 20-39.

Roberts, J. A. (1996) Green consumers in the 1990: profile and implications for advertising. Journal of Business Research, 36, 217-231.

Roberts, J. A., \& Bacon, D. R. (1997) Exploring the subtle relationship between environmental concern and ecologically conscious consumer behavior. Journal of Business Research, 40, 79-89.

Robert, J., Wessells, C., Donath, H., \& Asche, F. (2007) Measuring consumer preferences for Ecolabeled Seafood: An International Comparison. Journal of Agricultural and Resource Economics, 26(1), 20-39.

Schaefer, A., \& Crane, A. (2005) Addressing sustainability and consumption. Journal of Macromarketing, 25(1), 76-92.

Schlegelmilch, B. B., Bohlen, G. M., \& Diamantopoulos, A. (1996) The link between green purchasing decisions and measures of environmental consciousness. European Journal Marketing, $30(5), 35-55$.

Revista de Gestão Social e Ambiental - RGSA, São Paulo, v. 6, n. 1, p. 102-116, jan./abril 2012. 
Schwepker, C. H., \& Cornwell, T. B. (1991) An examination of ecologically concerned consumers and their intention to purchase ecologically packaged products. Journal of Public Policy and Marketing, 10(2), 77-101.

Shrum L. J., Mccarty, J.A., \& Lowrey T. M. (1995) Buyer characteristics of the green consumer and their implications for advertising strategy. Journal of Advertising, 24(2), 71-82.

Stone, G., Barnes, J. H., \& Montgomery, C. (1995) ECOSCALE: a scale for the measurement of environmentally responsible consumers. Psychology \& Marketing, 12, 595-612.

Straughan, R. D., \& Roberts, J. A. (1999) Environmental segmentation alternatives: a look at green consumer behavior in the new millennium. Journal of Consumer Marketing, 16(6), 558-575.

Tapon, F., \& Leighton, T. (1991) Green as strategy: lessons from the chemical industry. Strategic Management Society, 6-23, October.

Velter, A. N., Battistella, L. F., Grohmann, M. Z., \& Carpes, A. M. (2010) O estudo da sustentabilidade na administração: um levantamento dos "hot topics" publicados na última década. Anais do Seminário em Administração, São Paulo.

Wagner, S. A. (1997) Understanding green consumer behavior. Routledge, London.

Webster Jr., F. E. (1975) Determining the characteristics of the socially conscious consumer. Journal of Consumer Research, 2.

Data do recebimento do artigo: 10/11/2010

Data do aceite de publicação: 30/04/2012 